\title{
Rationale for using the velocity-time integral and the minute distance for assessing the stroke volume and cardiac output in point-of-care settings
}

\author{
Pablo Blanco * (1)
}

\begin{abstract}
Background: Stroke volume (SV) and cardiac output (CO) are basic hemodynamic parameters which aid in targeting organ perfusion and oxygen delivery in critically ill patients with hemodynamic instability. While there are several methods for obtaining this data, the use of transthoracic echocardiography (TTE) is gaining acceptance among intensivists and emergency physicians. With TTE, there are several points that practitioners should consider to make estimations of the SV/CO as simplest as possible and avoid confounders.

Main body: With TTE, the SV is usually obtained as the product of the left ventricular outflow tract (LVOT) crosssectional area (CSA) by the LVOT velocity-time integral (LVOTVTI); the CO results as the product of the SV and the heart rate (HR). However, there are important drawbacks, especially when obtaining the LVOT CSA and thus the impaction in the calculated SV and CO. Given that the LVOT CSA is constant, any change in the SV and CO is highly dependent on variations in the LVOT VTI; the HR contributes to CO as well. Therefore, the LVOTVTI aids in monitoring the SV without the need to calculate the LVOT CSA; the minute distance (i.e., SV $\times$ HR) aids in monitoring the CO. This approach is useful for ongoing assessment of the CO status and the patient's response to interventions, such as fluid challenges or inotropic stimulation. When the LVOTVTI is not accurate or cannot be obtained, the mitral valve or right ventricular outflow tract VTI can also be used in the same fashion as LVOTVTI. Besides its pivotal role in hemodynamic monitoring, the LVOTVTI has been shown to predict outcomes in selected populations, such as in patients with acute decompensated HF and pulmonary embolism, where a low LVOTVTI is associated with a worse prognosis.
\end{abstract}

Conclusion: The VTI and minute distance are simple, feasible and reproducible measurements to serially track the SV and $\mathrm{CO}$ and thus their high value in the hemodynamic monitoring of critically ill patients in point-of-care settings. In addition, the LVOT VTI is able to predict outcomes in selected populations.

Keywords: Ultrasonography, Echocardiography, Stroke volume, Cardiac output, Shock, Doppler, Point-of-care

\section{Background}

In applying any hemodynamic monitoring technique, flow parameters, such as the stroke volume (SV) and cardiac output $(\mathrm{CO})$, are key for targeting organ perfusion

\footnotetext{
*Correspondence: ohtusabes@gmail.com

Intensive Care Physician, Intensive Care Unit, Clínica Cruz Azul, 2651, 60 St., 7630 Necochea, Argentina
}

and oxygen delivery in patients with hemodynamic instability [1].

Since the SV and CO cannot be estimated reliably by clinical examination and routine assessment [2-4], several methods have been developed with the purpose to obtain these parameters in emergency and critically ill patients $[2,5]$. Among them, the pulmonary artery catheter (PAC) has long been the mainstay for hemodynamic monitoring of critically ill patients; however, the 
usefulness of this device has been questioned with regard to its unfavorable harms/benefits ratio and thus it is nowadays abandoned in most intensive care units (ICU) around the world [5-7]. Of note, the PAC is still considered the gold standard for comparison when other $\mathrm{SV} /$ $\mathrm{CO}$ monitors are tested.

An ideal SV/CO monitor should be non-invasive, continuous or rapidly repeatable, cost effective, reproducible, reliable during various physiological states and should also have a fast response time [2]. In this regard, the latest American Society of Echocardiography guideline recommends using both TTE and/or TEE for assessment of SV and $\mathrm{CO}$ in determining responses to medical and surgical therapies [8]. The consensus on circulatory shock and hemodynamic monitoring by the Task Force of the European Society of Intensive Care Medicine provides a similar recommendation as well [9]. Besides its recommendation, it is important to note that there is conflicting evidence regarding the interchangeability between echocardiography and the pulmonary artery catheter or $\mathrm{CO}$ monitors for estimating the SV or CO, with some studies not showing a good correlation between them [10-13] and many studies showing the opposite [14-18].

TTE shows clear advantages in comparison with other methods for monitoring the SV and CO, highlighting its non-invasiveness, low costs, bedside application, avoidance of ionizing radiation, repeatability and extensive availability. In addition, TTE offers the possibility to link the SV or CO status to its causative factor, for example hypovolemia, cardiac dysfunction, cardiac tamponade, acute cor pulmonale and/or a vasodilated circulation [19]. In comparison with TTE, TEE offers equal or better diagnostic and monitoring performances; however, its invasiveness, limited availability and cost factors are major limitations for its use. Also, a standard TEE probe cannot be kept in the patient for too long [2]. In current practice, the main indication of TEE for hemodynamic monitoring is in mechanically ventilated patients who lack suitable TTE windows [2].

The focus of this article is to revisit the rationale of monitoring the $\mathrm{SV}$ and $\mathrm{CO}$ using the velocity-time integral and the minute distance as assessed by TTE, addressing their limitations, feasibility and reliability, all aspects with concrete implications to intensivists and emergency physicians.

\section{Calculation of the SV and CO by TTE}

With TTE, the SV is usually obtained from the product of the LVOT cross-sectional area (CSA, in $\mathrm{cm}^{2}$ ) with the LVOT velocity-time integral or VTI (also known as stroke distance, in $\mathrm{cm}$ ) (Figs. 1 and 2). The LVOT CSA is derived from the LVOT diameter (LVOTd) using the formula $\pi r^{2}\left[3.1416 \times(\operatorname{LVOTd} / 2)^{2}\right]$, or its equivalent $(\text { LVOTd })^{2} \times 0.785$. The LVOTd is acquired from the parasternal long axis view, at a mid-systolic frame, measured from the inner edge to inner edge of the LVOT or eventually between the site of insertion of the right- and non-coronary aortic leaflets $[20,21]$. The LVOT VTI is obtained by tracing the envelope of the Doppler spectrum of LVOT systolic flow from the apical five- or threechamber view using pulsed-wave Doppler (PWD), with the sample volume placed within the LVOT, approximately at $1 \mathrm{~cm}$ distance to the aortic valve [20]. An optimal VTI is considered when alignment of the PWD sample volume is parallel to the subaortic flow and minimal spectral broadening is obtained [1]. The product of the SV and heart rate (HR) will yield the $\mathrm{CO}$ (in $\mathrm{L} / \mathrm{min}$ ).

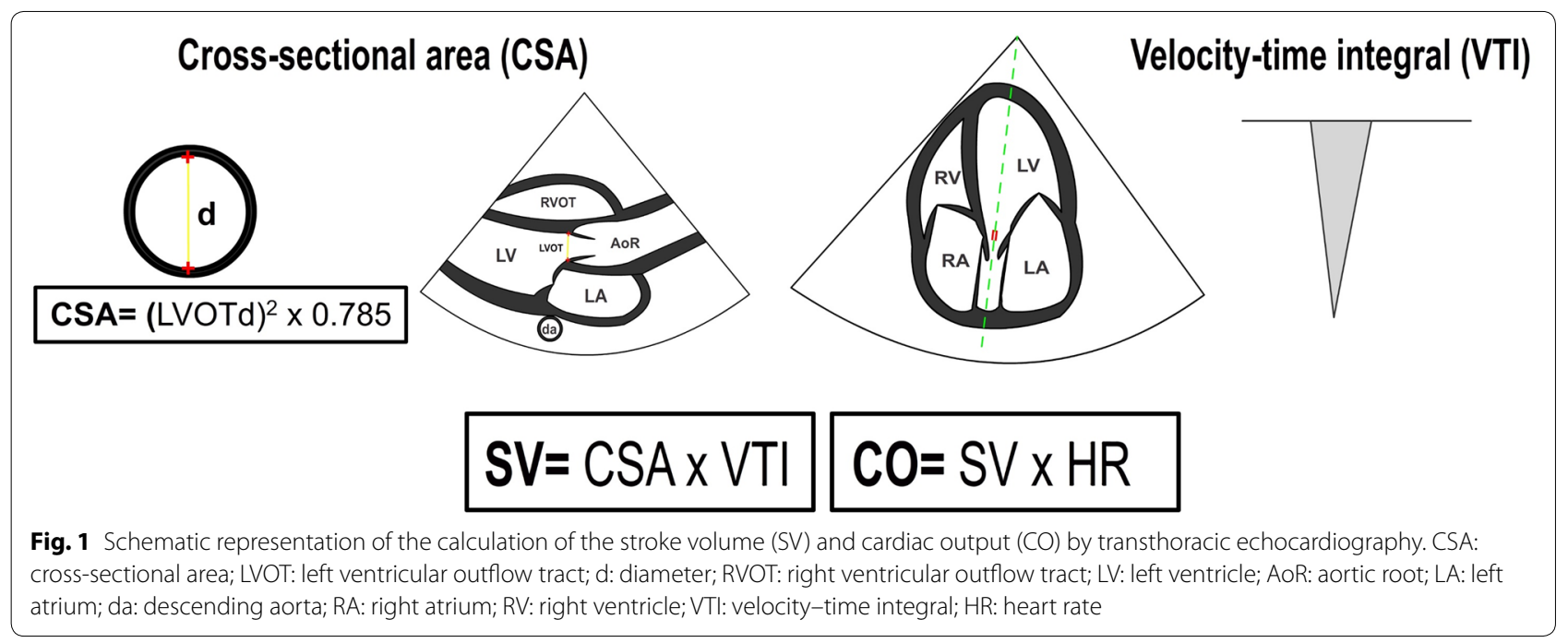




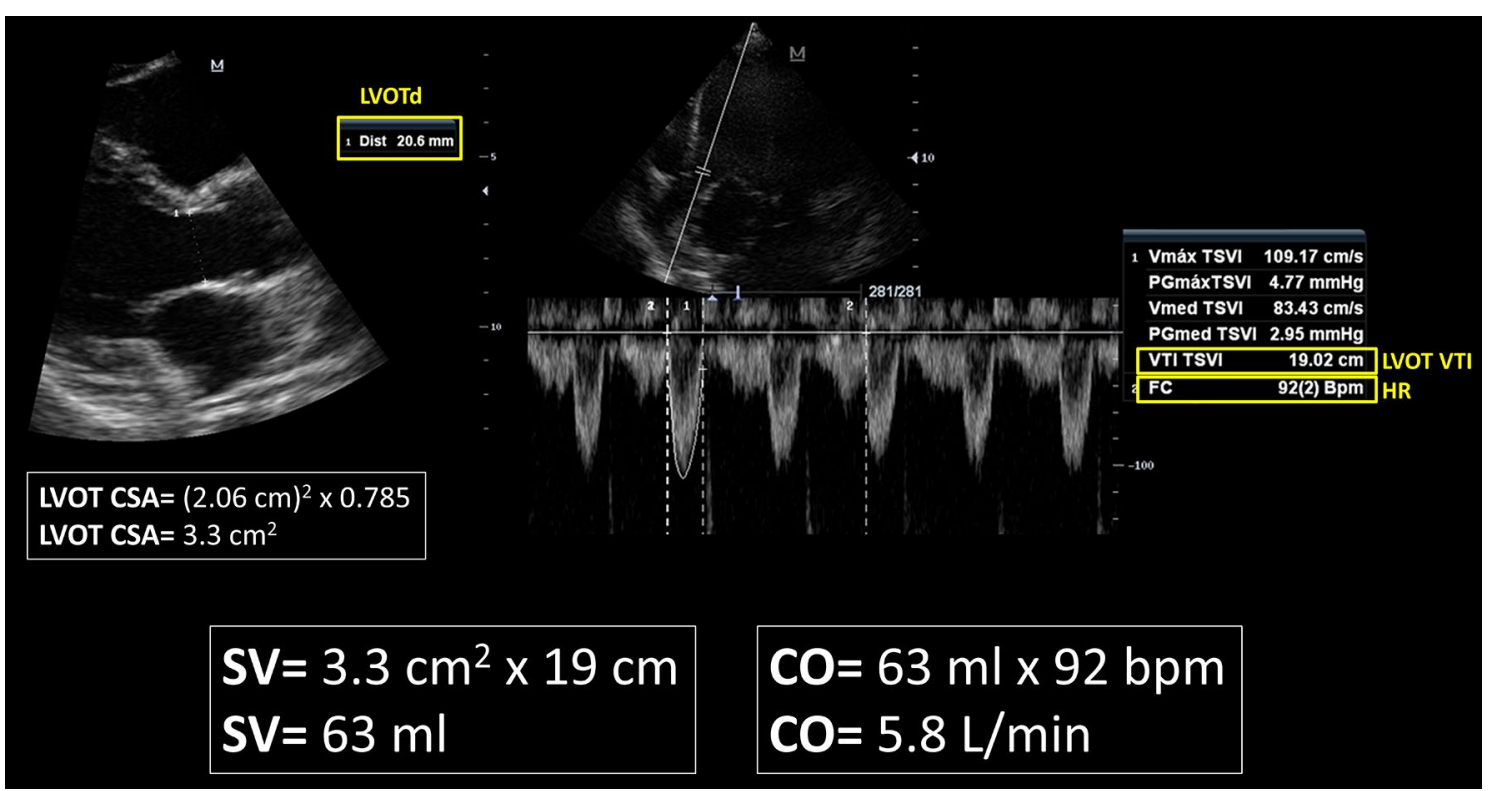

Fig. 2 Calculation of the stroke volume (SV) and cardiac output (CO) by transthoracic echocardiography in a critically ill patient without hemodynamic compromise. LVOT: left ventricular outflow tract; d: diameter; CSA: cross-sectional area; VTI: velocity-time integral; HR: heart rate

Based on the principle of mass conservation, which means that the $\mathrm{SV}$ and $\mathrm{CO}$ is the same across each valve or orifice in the absence of a significant valvular regurgitation or intracardiac shunt, other sites may also be used for measurement of the SV and CO, such as the right ventricular outflow tract (RVOT) or mitral valve (MV). In these cases, the CO is equal to RVOT VTI $\times$ RVOT $\mathrm{CSA} \times \mathrm{HR}$, and MV VTI $\times$ MV CSA $\times$ HR, respectively (Fig. 3) [19].

\section{Issues with the calculation of the SV and CO and the role of the VTI and minute distance}

According to the formula used to calculate the LVOT CSA, any measurement error in the LVOTd will be squared; therefore, there might be a large error in SV calculation. Unfortunately, measurement errors are not uncommon. For example, an LVOT of $1.8 \mathrm{~cm}$ gives a CSA of $2.5 \mathrm{~cm}^{2}$, while an LVOT $2 \mathrm{~mm}$ higher $(2 \mathrm{~cm})$ gives a CSA of $3.14 \mathrm{~cm}^{2}$. Assuming the same LVOT VTI for each CSA (e.g., $18 \mathrm{~cm}$ ), the SV in the first case is $45 \mathrm{~mL}$, while for the second case is $57 \mathrm{~mL}$ ( $26 \%$ higher than the former). As is noted, this large difference in calculation of the SV occurs with just a minimal difference in the measurement of the LVOTd.

Given that the LVOTd is essentially constant (as is the LVOT CSA), there is no need to measure it repeatedly, and this should be done once at baseline and then the same LVOT CSA is used for serial estimations of the SV and CO. When obtaining the LVOTd is not feasible, this can also be estimated based on a published equation
$[0.01 \times$ body height $(\mathrm{cm})+0.25][22]$. However, in practice, the LVOT CSA is rarely taken into account for tracking the SV or CO, since as is noted before, as the CSA is constant, any change in the SV must be the result of changes in LVOT VTI [21, 23, 24], while any change in the CO is due essentially to variations in the LVOT VTI, and also in the HR. Avoiding the CSA in the CO formula, the use of the VTI aids in assessing for serial changes in the SV while the minute distance, calculated as the VTI $\times \mathrm{HR}$, is useful to assess for serial changes in the $\mathrm{CO}$ (the latter is particularly useful when the HR varies significantly compared with the previous LVOT VTI measurement). Putting these concepts in practical examples:

Case 1: Patient with sepsis in whom fluid responsiveness is assessed (Fig. 4):

- Baseline measurements:

- $\operatorname{LVOTd}=2 \mathrm{~cm}\left(C S A=3.14 \mathrm{~cm}^{2}\right)$

- $\mathrm{LVOT} \mathrm{VTI}=13 \mathrm{~cm}$

- $\mathrm{SV}=41 \mathrm{~mL}$

- $\mathrm{HR}=80$ beats $/ \mathrm{min}$

- $\mathrm{CO}=3.3 \mathrm{~L} / \mathrm{min}$

- Minute distance $=1040 \mathrm{~cm} / \mathrm{min}$

- Measurements after a mini-fluid challenge $(200 \mathrm{~mL}$ of crystalloids):

- LVOTd and CSA = remain constant, equal to $3.14 \mathrm{~cm}^{2}$ 

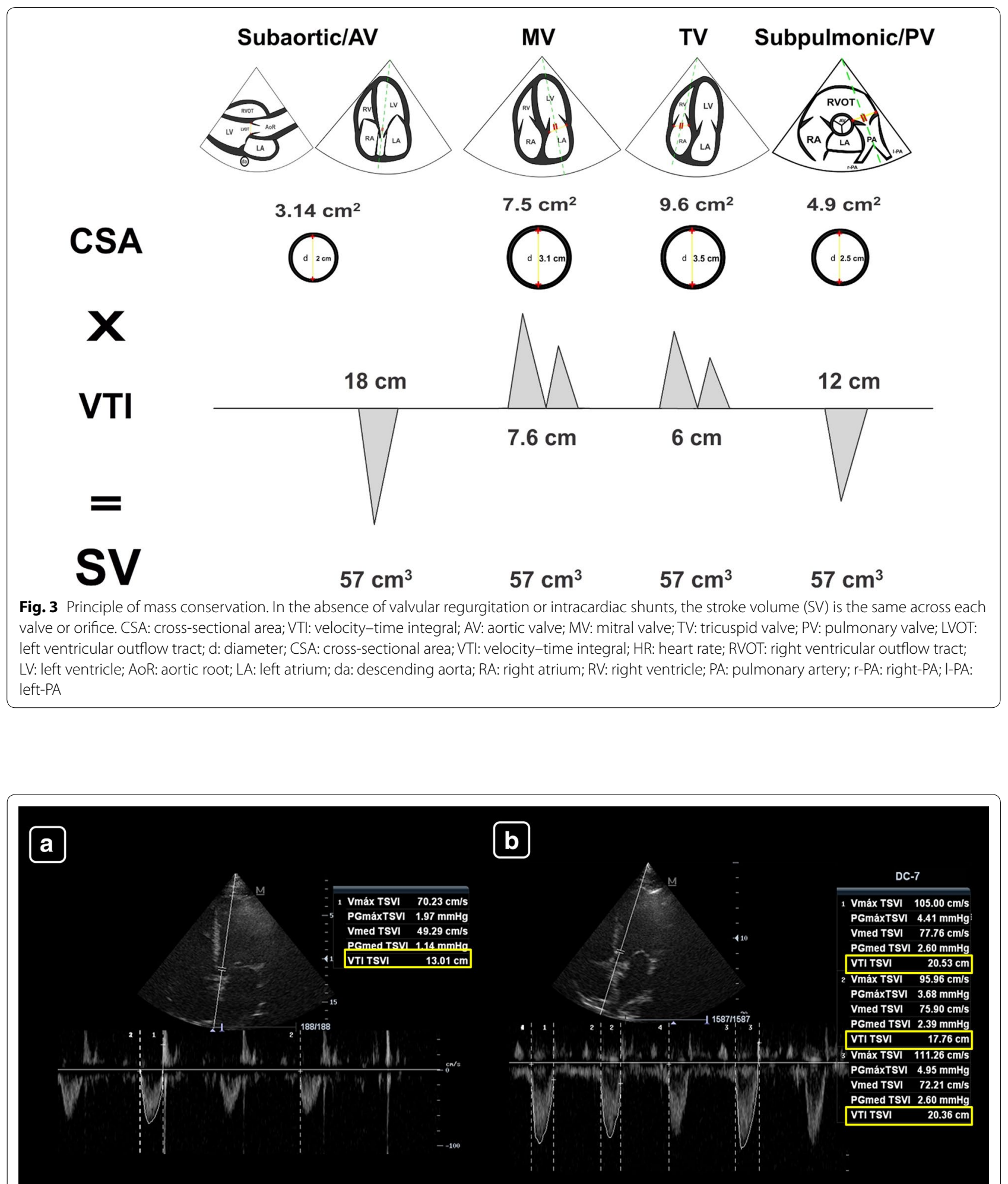

Fig. 4 Left ventricular tract velocity-time integral (LVOTVTI, yellow boxes) for assessing changes in the SV with treatments, in a patient with suspicion of hypovolemia. a Baseline. $\mathbf{b}$ After a fluid challenge. A $>15 \%$ increase in the VTI indicates a positive response to therapy, as seen in this case 
- LVOT VTI $=20 \mathrm{~cm}$ on average (increased $54 \%$ compared to baseline)

- $\mathrm{SV}=63 \mathrm{~mL}$ (increased 54\% compared to baseline)

- $\mathrm{HR}=80$ beats $/ \mathrm{min}$

- $\mathrm{CO}=5.1 \mathrm{~L} / \mathrm{min}$ (increased $54 \%$ compared to baseline)

- Minute distance $=1600 \mathrm{~cm} / \mathrm{min}$ (increased 54\% compared to baseline)

Case 2: Patient with cardiogenic shock (Fig. 5):

- Baseline measurements:

- LVOTd = cannot be obtained given that the PLAX was of inadequate quality

- $\mathrm{LVOT}$ VTI $=12.1 \mathrm{~cm}$

- $\mathrm{SV}=-$

- $\mathrm{HR}=104$ beats $/ \mathrm{min}$

- $\mathrm{CO}=-$

- Minute distance $=1248 \mathrm{~cm} / \mathrm{min}$

- Measurements on $10 \mathrm{ucg} / \mathrm{kg} / \mathrm{min}$ of dobutamine:

- LVOT d and CSA = remain constant, irrespective of its value

- $\mathrm{LVOT}$ VTI $=17.8 \mathrm{~cm}$ (increased $47 \%$ compared to baseline)

- $\mathrm{SV}=-$

- $\mathrm{HR}=111$ beats $/ \mathrm{min}$

- $\mathrm{CO}=-$

- Minute distance $=1976 \mathrm{~cm} / \mathrm{min}$ (increased 58\% compared to baseline)
As is shown, in the first case, the main datum indicating a change in the CO is the LVOT VTI, given that the LVOT CSA and the HR remain constant; in the second case, the LVOT VTI indicates a clear improvement in the SV (47\% compared to baseline) and the minute distance shows also a clear improvement in the $\mathrm{CO}$ (nearly $60 \%$ compared to baseline).

As absolute values, Goldman et al. [25] showed that when the HR is within the normal range, mean LVOT VTI values are about $20 \pm 3 \mathrm{~cm}(17-23 \mathrm{~cm})$; this indicates a normal SV and CO. When the HR is under $55 \mathrm{bpm}$, the LVOT VTI values must be higher than $18 \mathrm{~cm}$; otherwise, a low SV and $\mathrm{CO}$ are indicated and when the HR is higher than $95 \mathrm{bpm}$, LVOT VTI values must be lower than $22 \mathrm{~cm}$; otherwise, a high $\mathrm{SV}$ and $\mathrm{CO}$ are suggested (Fig. 6). However, more important than using isolated values of LVOT VTI or minute distance, changes of these parameters in response to treatments are of paramount interest. This concept is the basis of functional hemodynamic monitoring, which denotes that changes in cardiac function in response to treatments are more important than single static measurements [26].

\section{Practical application of the VTI and the minute distance}

The LVOT VTI has been used with success in several studies as a parameter for assessment of treatment responses, especially in the evaluation of fluid responsiveness [24, 26-29]. Mitral valve VTI was also used successfully for estimating volume responsiveness after a passive leg raising (PLR) test in one study [30]. From a rational point of view, the LVOT VTI (or other VTI) and the minute distance can be used to track changes in the SV and CO during the patient's follow-up and

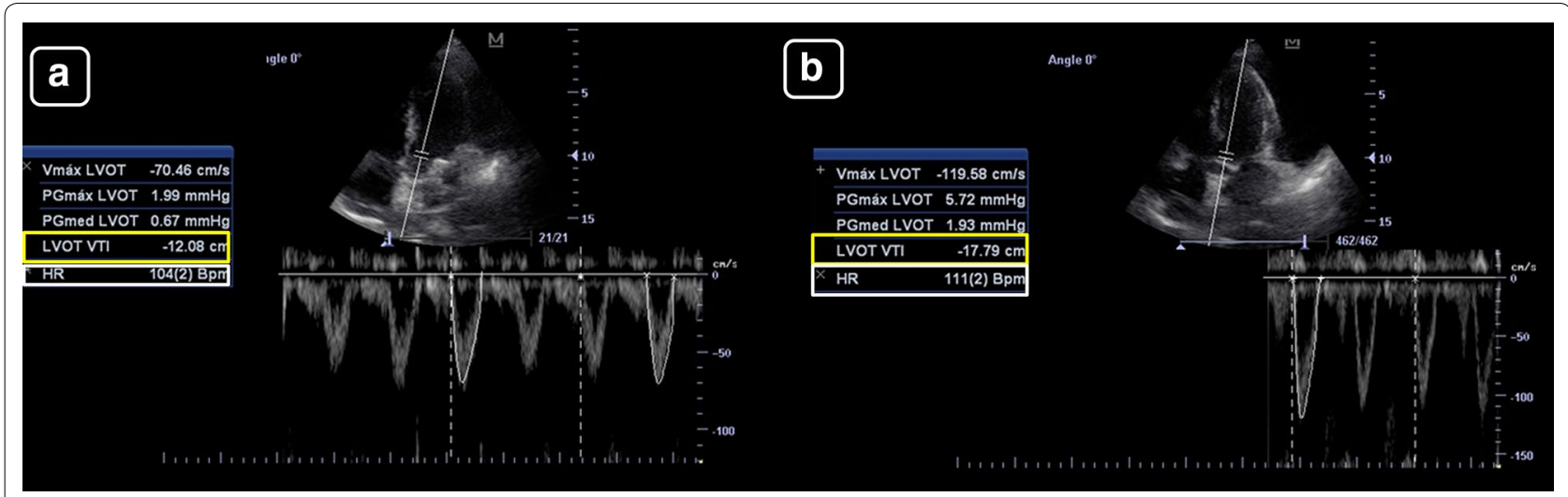

Fig. 5 Left ventricular tract velocity-time integral (LVOT VTI, yellow boxes) and minute distance (LVOT $x$ heart rate (HR, white boxes)) for assessing the stroke volume (SV) and cardiac output (CO) and their changes with treatments in a patient with cardiogenic shock. a Before treatment. b Receiving $10 \mathrm{ucg} / \mathrm{kg} / \mathrm{min}$ of dobutamine. There is a marked increase in the LVOTVTI and minute distance after treatment, indicating an improvement in the SV and CO 


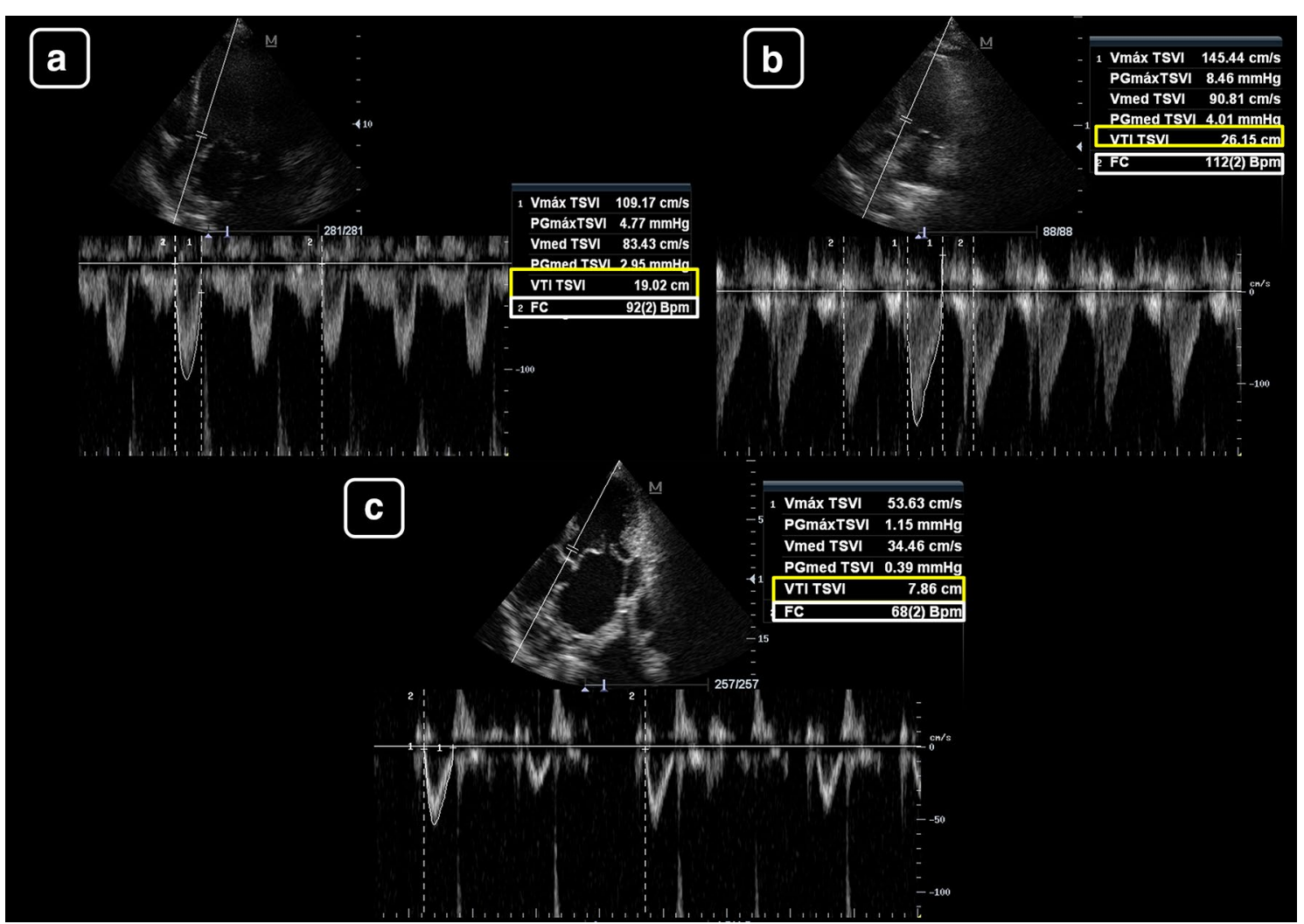

Fig. 6 Absolute values of the left ventricular tract velocity-time integral (LVOT VTI, yellow boxes) and minute distance (LVOT $\times$ heart rate (HR, white boxes)) for estimating the stroke volume (SV) and cardiac output (CO), respectively. a LVOTVTI of $19 \mathrm{~cm}$ and HR of 93 beats/min, indicating a normal SV and CO (the minute distance is $1748 \mathrm{~cm} / \mathrm{min}$ ). $\mathbf{b}$ LVOT VTI of $26 \mathrm{~cm}$ and HR of 113 beats/min, indicating a high SV and CO (the minute distance is $2912 \mathrm{~cm} / \mathrm{min}$ ). c LVOTVTI of $8 \mathrm{~cm}$ and HR of 68 beats/min, indicating a low SV and CO (the minute distance is $544 \mathrm{~cm} / \mathrm{min}$ )

to assess for the patient's response to the administered treatments, like fluid challenges, vasopressor therapy, inotropic support or relief of obstructive shock mechanisms. As a rule of thumb, an increase $>15 \%$ in the VTI after a treatment indicates a concrete response to this therapy or, to the contrary, its futility in case the VTI does not change accordingly [19]. With the PLR test, $a>12 \%$ increase in the VTI after a PLR has demonstrated a good accuracy for differentiating fluid responders from non-responders [27, 28]. Besides its value in hemodynamic monitoring, there is also a role of the LVOT VTI for predicting outcomes in selected populations, as seen in patients with acute decompensated heart failure [31] and in patients with acute pulmonary embolism [32], where a very low LVOT VTI $(<10 \mathrm{~cm})$ and a low LVOT VTI $(<15 \mathrm{~cm})$ is associated with worse prognosis, respectively. In other study, a low LVOT VTI $(<15 \mathrm{~cm})$ correlates with a low CO syndrome in patients with acute decompensated heart failure and predicts the use of inotropes [33].

\section{Limitations of the LVOT VTI}

The LVOT VTI is not reliable for estimating the SV/ $\mathrm{CO}$ when there is a moderate-to-severe aortic regurgitation (AR) and/or a subaortic obstruction (LVOTO, fixed and/or dynamic) [19] (Fig. 7). Dynamic LVOTO may be observed in extreme hypovolemia, asymmetric left ventricular septal hypertrophy (specially at a low preload and high inotropic stimulation), anterior myocardial infarctions with compensatory hyperdynamic basal segments of the interventricular septum [19] and in Takotsubo syndrome [34]. Dynamic LVOTO and AR produce high LVOT velocities/VTI and thus overestimated VTIs. Also, for assessing the response to treatments, there is no certainty regarding whether the changes in the LVOT VTI result from an increased SV or from an increased regurgitant volume (AR) or subaortic obstruction (LVOTO).

In some patients, obtaining apical views is problematic, such as in those with lung hyperinflation and/or obese individuals. 


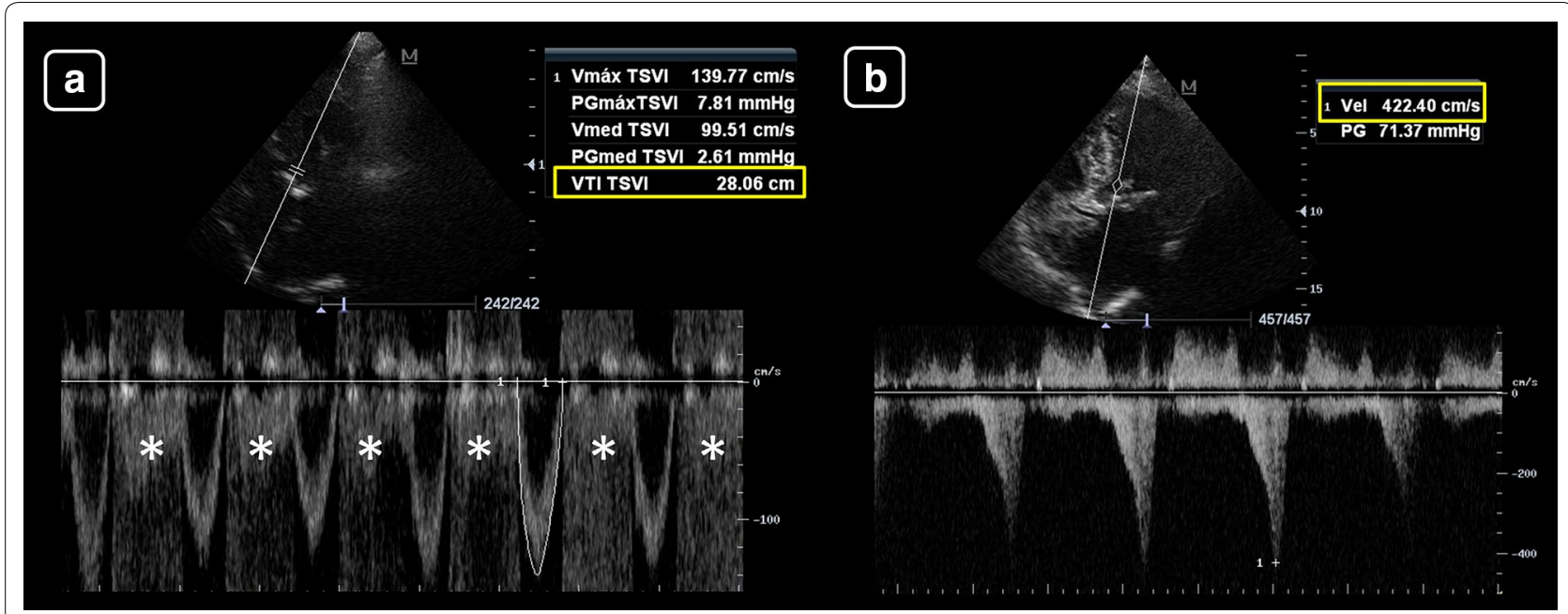

Fig. 7 a Left ventricular outflow tract velocity-time integral (LVOTVTI) in severe aortic regurgitation. LVOTVTI showed high values (yellow box), overestimating the stroke volume. Asterisks: aliased spectral Doppler signal of aortic regurgitation. $\mathbf{b}$ LVOT VTI in dynamic LVOT obstruction, obtained with continuous wave Doppler (pulsed-wave Doppler waveforms were aliased). The spectral Doppler showed high blood flow peak velocities $(4.2 \mathrm{~m} / \mathrm{s}$, yellow box) and a long time-to-peak signal. This situation can be seen in severe hypovolemia, hypertrophic cardiomyopathy, anterior myocardial infarctions with hypercontractile basal segments and in Takotsubo syndrome

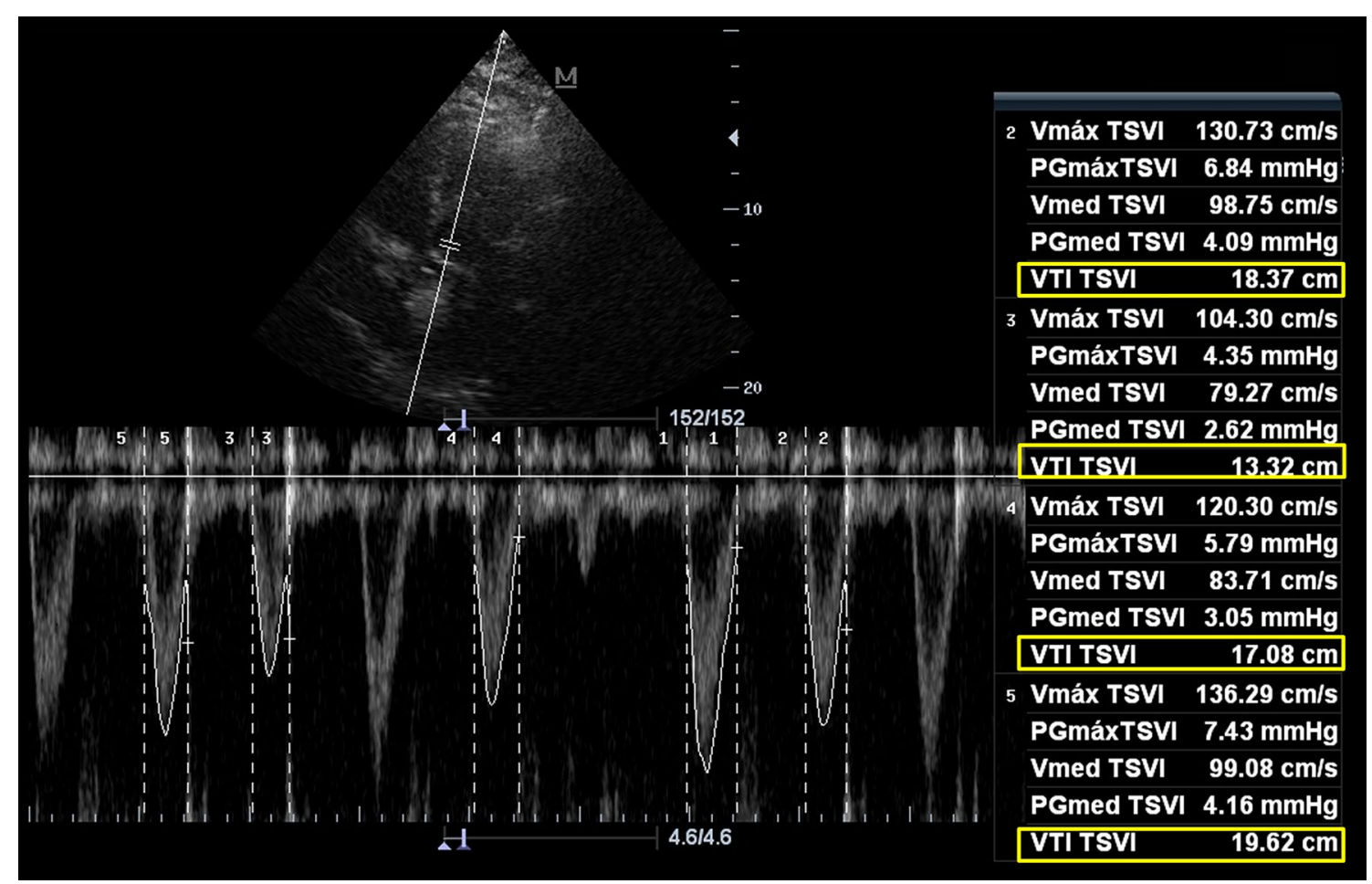

Fig. 8 Averaging several left ventricular tract velocity-time integral (LVOTVTI, (yellow boxes)) for obtaining a mean VTI in the context of atrial fibrillation

A practical problem often observed is related to maintain the probe in the same position for measuring repeatedly the VTI with accuracy, particularly when using the
PLR test. For this indication, the Probefix ${ }^{\circledR}$ (i.e., an external ultrasound probe holder strapped to the patient) may have a potential value; however, in a small study using 
this device, there were no advantages compared with the manually recorded SV and CO using TTE [35].

As a practical issue, obtaining the LVOT VTI often requires several key strokes and may be time-consuming. As technology advances, manufacturers provide a way to do that with less effort and saving time, particularly with the utilization of the auto-VTI ${ }^{\circledR}$ software. This software has been tested in an animal (piglets) experimental model of hemorrhagic shock and demonstrated a better correlation with the $\mathrm{CO}$ obtained by thermodilution when compared with the conventional echocardiography technique [36].

Finally, a common issue for the accurate VTI determination is the presence of arrhythmias, especially atrial fibrillation and frequent extrasystoles, since different filling times result in beat-to-beat VTI variability. In these cases, it is recommended to average at least five VTIs in order to obtain a more accurate VTI value (Fig. 8) [23, 37].

\section{Feasibility and reliability for the VTI in point-of-care settings}

Regarding feasibility, in the study carried out by Bergenzaun et al. [38] that evaluated echocardiographic parameters to assess the LV function in 50 patients with shock and mechanical ventilation, the LVOT VTI was obtained in $95 \%$ of all possible examinations and repeatability was high. The study carried out by Dinh et al. [1] showed that emergency physicians can accurately measure SV and CO using TTE in the emergency department. In this study including 97 patients, an optimal LVOT VTI was obtained in $78.4 \%$ of patients. More recently, McGregor et al. showed a feasibility of 78.7\% for the LVOT VTI [29].

Regarding reliability, if expected physiologic responses range between increments of at least $15 \%$ in VTI after an intervention, intra- and interobserver variability for the measurement of VTI must be lower than these values, otherwise, the margin of error may exceed the patient's physiologic response. Regarding this point, the reported intra- and interobserver variabilities are low among studies (ranging between 3 and 8\%) [16, 24, $29,38]$. In a recent study, the lowest smallest change for the LVOT VTI (i.e., the smallest change that can be considered as significant and not related to the imprecision of the method or the variability of the parameter) was found to be $<5 \%$ for intra-examinations (i.e., without removing the probe from the chest wall), while it averages $11 \%$ for inter-examinations (i.e., removing the probe from the chest wall) [37].

All these data indicate that the LVOT VTI is a feasible and reliable parameter for assessing patients with hemodynamic compromise in the ICU or emergency department, particularly when measured by experienced TTE operators.

\section{Conclusions}

Serial assessment of the SV and CO can be done measuring the VTI (LVOT, MV or RVOT) and calculating the minute distance, without the need to know the CSA. Changes in the VTI directly reflect modifications in SV while changes in the minute distance reflect modifications in the $\mathrm{CO}$, and this data may aid in the ongoing assessment of the $\mathrm{CO}$ status and the patient's response to treatments.

\section{Abbreviations}

SV: Stroke volume; CO: Cardiac output; LVOT: Left ventricular outflow tract; VTI: Velocity-time integral; CSA: Cross-sectional area; d: Diameter; TTE: Transthoracic echocardiography; HR: Heart rate; TEE: Transesophagic echocardiography; PWD: Pulsed-wave Doppler; RVOT: Right ventricular outflow tract; MV: Mitral valve; ICU: Intensive care unit; LVOTO: Left ventricular outflow tract obstruction; PAC: Pulmonary artery catheter; PLR: Passive leg raising.

\section{Acknowledgements \\ This work has not been presented at any conferences.}

\section{Authors' contributions}

The author PB wrote the manuscript, and edited the figures. The author read and approved the final manuscript.

\section{Funding}

The author does not receive any funding.

\section{Availability of data and materials \\ Not applicable.}

\section{Ethics approval and consent to participate}

All procedures performed in studies involving human participants were in accordance with the ethical standards of the institutional and/or national research committee and with the 1964 Helsinki declaration and its later amendments or comparable ethical standards.

\section{Consent for publication}

Not applicable.

\section{Competing interests}

The author declares no competing interests.

Received: 22 November 2019 Accepted: 15 April 2020

Published online: 21 April 2020

\section{References}

1. Dinh VA, Ko HS, Rao R, Bansal RC, Smith DD, Kim TE, Nguyen HB (2012) Measuring cardiac index with a focused cardiac ultrasound examination in the ED. Am J Emerg Med 30:1845-1851

2. Mehta Y, Arora D (2014) Newer methods of cardiac output monitoring. World J Cardiol 6(9):1022-1029

3. Iregui MG, Prentice D, Sherman G, Schallom L, Sona C, Kollef MH (2003) Physicians' estimates of cardiac index and intravascular volume based on clinical assessment versus transesophageal Doppler measurements obtained by critical care nurses. Am J Crit Care 12:336-342

4. Johnson A, Ahrens T (2015) Stroke volume optimization: the new hemodynamic algorithm. Crit Care Nurse 35(1):11-27

5. Nguyen HB, Banta DP, Stewart G, Kim T, Bansal R, Anholm J, Wittlake WA, Corbett SW (2010) Cardiac index measurements by transcutaneous 
Doppler ultrasound and transthoracic echocardiography in adult and pediatric emergency patients. J Clin Monit Comput 24(3):237-247

6. Marik PE (2013) Obituary: pulmonary artery catheter 1970 to 2013. Ann Intensive Care 3:38

7. van Lelyveld-Haas LE, van Zanten AR, Borm GF, Tjan DH (2008) Clinical validation of the non-invasive cardiac output monitor USCOM-1A in critically ill patients. Eur J Anaesthesiol 25:917-924

8. Porter TR, Shillcutt SK, Adams MS, Desjardins G, Glas KE, Olson JJ, Troughton RW (2015) Guidelines for the use of echocardiography as a monitor for therapeutic intervention in adults: a report from the American Society of Echocardiography. J Am Soc Echocardiogr 28(1):40-56

9. Cecconi M, De Backer D, Antonelli M, Beale R, Bakker J, Hofer C, Jaeschke R, Mebazaa A, Pinsky MR, Teboul JL, Vincent JL, Rhodes A (2014) Consensus on circulatory shock and hemodynamic monitoring. Task force of the European Society of Intensive Care Medicine. Intensive Care Med 40(12):1795-1815

10. Blancas R, Martínez-González Ó, Ballesteros D, Núñez A, Luján J, Rodríguez-Serrano D, Hernández A, Martínez-Díaz C, Parra CM, Matamala BL, Alonso MA, Chana M (2019) Lack of correlation between left ventricular outflow tract velocity time integral and stroke volume index in mechanically ventilated patients. Med Intensiva 43(2):73-78

11. Wetterslev M, Møller-Sørensen H, Johansen RR, Perner A (2016) Systematic review of cardiac output measurements by echocardiography vs. thermodilution: the techniques are not interchangeable. Intensive Care Med 42(8):1223-1233

12. Staer-Jensen H, Sunde K, Nakstad ER, Eritsland J, Andersen GØ (2018) Comparison of three haemodynamic monitoring methods in comatose post cardiac arrest patients. Scand Cardiovasc J 52(3):141-148

13. Souto Moura T, Aguiar Rosa S, Germano N, Cavaco R, Sequeira T, Alves M, Papoila AL, Bento L (2018) The accuracy of PiCCO ${ }^{\circledR}$ in measuring cardiac output in patients under therapeutic hypothermia: comparison with transthoracic echocardiography. Med Intensiva 42(2):92-98

14. Mercado P, Maizel J, Beyls C, Titeca-Beauport D, Joris M, Kontar L, Riviere A, Bonef O, Soupison T, Tribouilloy C, de Cagny B, Slama M (2017) Transthoracic echocardiography: an accurate and precise method for estimating cardiac output in the critically ill patient. Crit Care 21(1):136

15. Zhang Y, Wang Y, Shi J, Hua Z, Xu J (2019) Cardiac output measurements via echocardiography versus thermodilution: a systematic review and meta-analysis. PLoS ONE 14(10):e0222105

16. Villavicencio C, Leache J, Marin J, Oliva I, Rodriguez A, Bodí M, Soni NJ (2019) Basic critical care echocardiography training of intensivists allows reproducible and reliable measurements of cardiac output. Ultrasound J 11(1):5

17. Dark PM, Singer M (2004) The validity of trans-esophageal Doppler ultrasonography as a measure of cardiac output in critically ill adults. Intensive Care Med 30(11):2060-2066

18. Laupland KB, Bands CJ (2002) Utility of esophageal Doppler as a minimally invasive hemodynamic monitor: a review. Can J Anaesth 49(4):393-401

19. Blanco P, Aguiar FM, Blaivas M (2015) Rapid ultrasound in shock (RUSH) velocity-time integral: a proposal to expand the rush protocol. J Ultrasound Med 34(9):1691-1700

20. British Society of Echocardiography Education Committee. A minimum dataset for a standard transthoracic echocardiogram. http://www.bsech o.org/media/71250/tte_ds_sept_2012.pdf. Accessed 15 Dec 2019

21. Armstrong $W$, Ryan $T$ (2010) Hemodynamics. In: Armstrong W, Ryan $T$ (eds) Feigenbaum's echocardiography. Lippincott Williams \& Wilkins, Philadelphia, pp 217-240

22. Nidorf SM, Picard MH, Triulzi MO, Thomas JD, Newell J, King ME, Weyman AE (1992) New perspectives in the assessment of cardiac chamber dimensions during development and adulthood. J Am Coll Cardiol 19(5):983-988

23. Cholley BP (2011) Measurement of stroke volume and cardiac output using echocardiography and Doppler. In: de Backer D, Cholley BP, Slama $M$, Vieillard-Baron A, Vignon P (eds) Hemodynamic monitoring using echocardiography in the critically ill. Springer, Berlin, pp 51-60
24. Muller L, Toumi M, Bousquet PJ, Riu-Poulenc B, Louart G, Candela D, Zoric L, Suehs C, de La Coussaye JE, Molinari N, Lefrant JY, AzuRéa Group (2011) An increase in aortic blood flow after an infusion of $100 \mathrm{ml}$ colloid over 1 minute can predict fluid responsiveness: the mini-fluid challenge study. Anesthesiology 115(541-7):25

25. Goldman JH, Schiller NB, Lim DC, Redberg RF, Foster E (2001) Usefulness of stroke distance by echocardiography as a surrogate marker of cardiac output that is independent of gender and size in a normal population. Am J Cardiol 87:499-502

26. Monnet $X$, Teboul JL (2013) Assessment of volume responsiveness during mechanical ventilation: recent advances. Crit Care 17(2):217

27. Maizel J, Airapetian N, Lorne E, Tribouilloy C, Massy Z, Slama M (2007) Diagnosis of central hypovolemia by using passive leg raising. Intensive Care Med 33:1133-1138

28. Lamia B, Ochagavia A, Monnet X, Chemla D, Richard C, Teboul JL (2007) Echocardiographic prediction of volume responsiveness in critically ill patients with spontaneously breathing activity. Intensive Care Med 33:1125-1132

29. McGregor D, Sharma S, Gupta S, Ahmad S, Godec T, Harris T (2019) Emergency department non-invasive cardiac output study (EDNICO): a feasibility and repeatability study. Scand J Trauma Resusc Emerg Med 27(1):30

30. Bou Chebl R, Abou Dagher G, Wuhantu J, Bachir R, Carnell J (2018) Mitral valve velocity time integral and passive leg raise as a measure of volume responsiveness. Crit Ultrasound J 10(1):32

31. Tan C, Rubenson D, Srivastava A, Mohan R, Smith MR, Billick K, Bardarian S, Thomas Heywood J (2017) Left ventricular outflow tract velocity time integral outperforms ejection fraction and Doppler-derived cardiac output for predicting outcomes in a select advanced heart failure cohort. Cardiovasc Ultrasound 15(1):18

32. Yuriditsky E, Mitchell OJ, Sibley RA, Xia Y, Sista AK, Zhong J, Moore WH, Amoroso NE, Goldenberg RM, Smith DE, Jamin C, Brosnahan SB, Maldonado TS, Horowitz JM (2019) Low left ventricular outflow tract velocity time integral is associated with poor outcomes in acute pulmonary embolism. Vasc Med. https://doi.org/10.1177/1358863X19880268 (Epub ahead of print)

33. Chinen D, Fujino M, Anzai T, Kitakaze M, Goto Y, Ishihara M, Shimizu W, Yokoyama H, Yasuda S, Ogawa H (2013) Left ventricular outflow tract velocity time integral correlates with low cardiac output syndrome in patients with acute decompensated heart failure [abstract]. Eur Heart $\rfloor$ 34(suppl):P4249

34. Kawaji T, Shiomi H, Morimoto T, Tazaki J, Imai M, Saito N, Makiyama T, Shizuta S, Ono K, Kimura T (2015) Clinical impact of left ventricular outflow tract obstruction in Takotsubo cardiomyopathy. Circ J 79(4):839-846

35. Blans MJ, Bosch FH, van der Hoeven JG (2019) The use of an external ultrasound fixator (Probefix) on intensive care patients: a feasibility study. Ultrasound J 11(1):26

36. Bobbia X, Muller L, Claret PG, Vigouroux L, Perez-Martin A, de La Coussaye JE, Lefrant JY, Louart G, Roger C, Markarian T (2019) New echocardiographic tool for cardiac output evaluation: an experimental study. Shock 52(4):449-455

37. Jozwiak M, Mercado P, Teboul JL, Benmalek A, Gimenez J, Dépret F, Richard C, Monnet X (2019) What is the lowest change in cardiac output that transthoracic echocardiography can detect? Crit Care 23(1):116

38. Bergenzaun L, Gudmundsson P, Öhlin H, Düring J, Ersson A, Ihrman L, Willenheimer R, Chew MS (2011) Assessing left ventricular systolic function in shock: evaluation of echocardiographic parameters in intensive care. Crit Care 15(4):R200

\section{Publisher's Note}

Springer Nature remains neutral with regard to jurisdictional claims in published maps and institutional affiliations. 